\title{
Rituximab use for refractory anti-HMGCR immune-mediated necrotizing myopathy: A case report
}

\author{
Sonia Gupta ${ }^{1, *}$, Amandeep Rakhra ${ }^{2}$, Vinay Thallapally ${ }^{1}$, Joseph Nahas ${ }^{1}$ \\ ${ }^{1}$ Department of Internal Medicine, Creighton University, Omaha, Nebraska, USA; \\ ${ }^{2}$ Department of Rheumatology, Dartmouth-Hitchcock Medical Center, Lebanon, New Hampshire, USA.
}

SUMMARY Immunosuppression is the cornerstone therapy for anti-3-hydroxy-3-methylglutaryl-coenzyme A reductase (anti-HMGCR) myopathy. Typical immunosuppressants such as corticosteroids, methotrexate, and azathioprine have been used in conjunction with removal of the offending agent, yet the use of rituximab is more limited in this type of myopathy. Reported here is a case of a patient who responded well to rituximab (RTX) after the standard immunosuppressants had failed. This case illustrates the importance of further studies to evaluate the role of RTX in anti-HMGCR myopathy.

Keywords anti-HMGCR, immune-mediated necrotizing myopathy, immunosuppressants, rituximab

\section{Introduction}

Immune-mediated necrotizing myopathy (IMNM) is an autoimmune condition resulting from the direct or indirect injury of myofibers by the immune system. Anti-3-hydroxy-3-methylglutaryl-coenzyme A reductase (anti-HMGCR) myopathy is a subtype of IMNM and was first described in 2010 (1). It has been seen both in statin-naïve and statin-exposed patients, with a prevalence of 1-2 cases per million in those who are statin-exposed (2). It presents with muscle weakness, myalgia, and elevated serum creatine phosphokinase with a mean age of 55 years. Studies have found that statin-naïve patients were younger than statin-exposed patients (3). There are currently no guidelines for the management of anti-HMGCR myopathy. Immunosuppressants are the cornerstone of therapy, but very few studies regarding the use of rituximab (RTX) have been published - all with varying responses (4). Described here is a case of antiHMGCR-associated IMNM refractory to conventional immunosuppressants but responsive to RTX.

\section{Case Report}

A 61-year-old female with a history of primary hypercholesterolemia, hypothyroidism, and hypertension presented with rapidly progressing proximal upper and lower muscle extremity weakness for seven days. Her symptoms were associated with muscle pain and slowly progressive dysphagia to both solids and liquids. Her medication history included taking pravastatin for two years. A physical examination revealed a grade of $3 / 5$ on the Medical Research Council (MRC) Scale for Muscle Strength in the proximal upper and lower extremities bilaterally. Laboratory tests including a complete blood count, renal function test, and inflammatory markers (erythrocyte sedimentation rate (ESR) and C-reactive protein (CRP)) were normal. Her creatinine kinase level was 15,000 U/L (normal, 26-192 U/L), her myoglobin level was higher than 1,000 (normal, 9-83ng/mL), and aspartate aminotransferase (309 U/L; normal: 10-40 U/ L) and alanine aminotransferase (691 U/L; normal: 12$78 \mathrm{U} / \mathrm{L}$ ) were elevated (Table 1). Differentials were polymyositis, statin-induced myopathy, paraneoplastic myopathy, and anti-signal recognition particle-associated myopathy.

Liver ultrasound was normal. Statin was discontinued and oral prednisone at a dose of $60 \mathrm{mg}$ was started since IMNM was suspected. An initial rheumatologic workup for autoimmune myopathies was negative including rheumatoid factor, antinuclear antibody, and anti-myositis antibodies including anti Jo1, anti Ro, anti-signal recognition particle, anti-mi-2, acetylcholine receptor antibody, and muscle-specific tyrosine kinase antibody. Pan computed tomography including the chest, abdomen, and pelvis did not reveal evidence of a malignancy. Magnetic resonance imaging of the right lower extremity revealed diffuse patchy muscular atrophy throughout the thigh with extensive patchy muscle edema involving the anterior medial and posterior compartments. The right quadriceps was biopsied, an electromyogram and antiHMGCR antibodies were ordered, and pending biopsy results the patient was discharged on $60 \mathrm{mg}$ of steroids. 
Table 1. Laboratory results from the initial visit and subsequent visits

\begin{tabular}{|c|c|c|c|c|c|c|c|c|c|}
\hline Laboratory tests & $\begin{array}{l}\text { Normal } \\
\text { reference } \\
\text { values }\end{array}$ & On admission & $\begin{array}{l}28 \text { days } \\
\text { On the steroid } \\
\text { azathioprine }\end{array}$ & $\begin{array}{c}42 \text { days } \\
\text { Azathioprine } \\
\text { discontinued } \\
\text { and rituximab } \\
\text { initiated }\end{array}$ & $\begin{array}{c}56 \\
\text { days }\end{array}$ & $\begin{array}{c}84 \\
\text { days }\end{array}$ & $\begin{array}{c}112 \\
\text { days }\end{array}$ & $\begin{array}{c}168 \\
\text { days }\end{array}$ & $\begin{array}{c}365 \\
\text { days }\end{array}$ \\
\hline Erythrocyte Sedimentation Rate & $0 / 15 \mathrm{~mm} / \mathrm{hour}$ & 7 & & & & & & & \\
\hline C-Reactive Protein & $\leq 9 \mathrm{mg} / \mathrm{L}$ & $<2.90$ & $<2.90$ & & & & & & \\
\hline Creatinine Kinase & $26-192 \mathrm{U} / \mathrm{L}$ & 15,000 & 2,040 & 1,300 & 989 & 837 & 132 & 114 & 96 \\
\hline Aspartate Aminotransferase & $10-40 \mathrm{U} / \mathrm{L}$ & 309 & 86 & & 47 & 29 & 20 & 20 & \\
\hline Alanine Aminotransferase & $12-78 \mathrm{U} / \mathrm{L}$ & 691 & 447 & & 151 & 49 & 38 & 36 & \\
\hline Total Bilirubin & $0-1.5 \mathrm{mg} / \mathrm{dL}$ & 0.4 & 0.4 & & 0.6 & 0.4 & 0.4 & 0.3 & \\
\hline Myoglobin & $9-83 \mathrm{ng} / \mathrm{mL}$ & $>1000$ & & & & & & & \\
\hline Thyroid-stimulating Hormone & $0.4-5 \mathrm{uIU} / \mathrm{mL}$ & 2.124 & & & 2.330 & & & & \\
\hline Carcinoembryonic Antigen & & Negative & & & & & & & \\
\hline Myositis-specific Antibodies & & Negative & & & & & & & \\
\hline CA-19-9 & & Negative & & & & & & & \\
\hline CA 125 & & Negative & & & & & & & \\
\hline Rheumatoid Factor & $<15 \mathrm{IU} / \mathrm{mL}$ & $<10$ & & & & & & & \\
\hline Antinuclear Antibody & & Negative & & & & & & & \\
\hline Aldolase & $1.5-8 \mathrm{U} / \mathrm{L}$ & 135 & 63.6 & & 6.1 & & & & \\
\hline
\end{tabular}

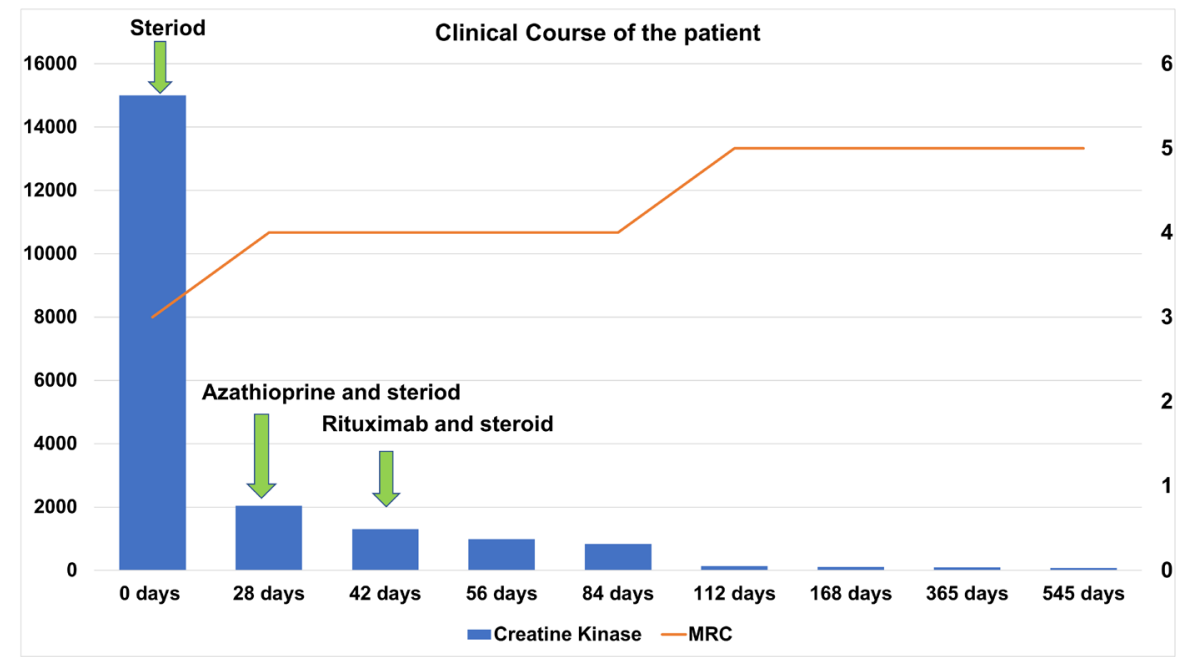

Figure 1. Clinical course after rituximab was initiated and muscle strength recovered to the baseline level after 3 months of rituximab therapy: CK levels and muscle strength on the MRC scale (data points). Less muscle strength in the hip flexors was noted on admission according to the MRC scale.

During her 4-week follow up in the rheumatology clinic, she was still complaining of weakness. Her upper arms and bilateral hip flexors were MHC grade $4 / 5$. Her repeat creatine kinase (CK) level was around 2,000 U/L. The quadriceps biopsy revealed scattered myofiber atrophy, degeneration, regeneration, necrosis, and myophagocytosis suggestive of necrotizing myopathy. Electromyography (EMG) was performed and revealed myositis, and anti-HMG CoA reductase antibodies were positive, with a titer of 99 (normal $<20$ units) suggestive of anti-HMGCR-induced myopathy. The patient was subsequently started on azathioprine (AZA) $50 \mathrm{mg}$ daily.

Fifteen days later, patient complained of nausea and did not want to continue taking AZA. She still had weakness in her upper extremities. Her aspartate aminotransferase level was $82 \mathrm{U} / \mathrm{L}$, her alanine aminotransferase level was $269 \mathrm{U} / \mathrm{L}$, and her CK level was $1,300 \mathrm{U} / \mathrm{L}$. The steroid dose was decreased to 30 $\mathrm{mg}$, AZA was stopped, and a plan was formulated to start the patient on an RTX intravenous infusion 1,000 $\mathrm{mg} / \mathrm{m}^{2}$ with a second dose repeated 2 weeks later. Four weeks after RTX was initiated, her strength improved in her shoulders, fingers and toes, and proximal hip flexors. Her RTX dosage was then decreased to $375 \mathrm{mg} / \mathrm{mm}^{2}$ given once every 3 months. Remission was deemed to have been achieved after six doses of RTX since the patient's CK level and aldolase level were consistently normal and she was asymptomatic. The patient was also started on alirocumab for hyperlipidemia. The patient responded well to RTX within 4 weeks of treatment initiation and went into complete remission within 3 months of initiation of RTX, with normal CK levels and no relapse (Figure 1). She subsequently tolerated her maintenance therapy for one year with no complications. She is currently symptom-free. 


\section{Discussion}

Anti-HMGCR myopathy is a rare subtype of severe necrotizing autoimmune myopathy (5-7). There are few prospective studies and clinical trials in the literature to guide therapy for this specific myopathy. The regimen mainly depends on disease severity and a discussion between the rheumatologist and the patient. Prompt discontinuation of statin therapy and immunosuppressants are the initial treatment for statininduced IMNM. Common immunosuppressive that have been shown to be effective are corticosteroids, intravenous immunoglobulin (IVIG), AZA, methotrexate (MTX), and mycophenolate mofetil $(5,9,10)$. MTX or IVIG have been used for refractory cases, with both resulting in improvement $(3,10-12)$. The postulated mechanism for anti-HMGCR myopathy includes autoimmunity against HMGCR; since B cells play a big role in autoantibody production, $\mathrm{B}$ cell depletion would appear to be an effective treatment strategy for the condition (2). Experience with RTX is limited. In 2016, the European Neuromuscular Centre (ENMC) convened a workshop on the definition and treatment of IMNM for anti-HMGCR myopathy. The experts reached the consensus that patients with anti-HMGCR myopathy should be treated with both corticosteroids as well as a second-line agent either immediately or within 1 month of presentation, depending on the severity of disease and the response to steroid treatment. They suggested RTX as a possible alternative and indicated that it could be combined with MTX in severe cases (13).

There are very few studies on RTX response in refractory anti-HMGCR IMNM. Landon-Cardinal et al. conducted a retrospective study in which 9 out of 46 patients with anti-HMGCR IMNM received RTX after failing to respond to an average of three other immunosuppressants. All patients presented with proximal muscle weakness and a mean CK level 1,602 IU/L prior to RTX. Three out of nine patients on RTX had improved muscle strength and improved MRI findings. The most striking effect of RTX was seen in two statin-naïve younger patients, and the response was seen in the first month after starting the therapy. RTX was also helpful in weaning two patients off of corticosteroids and IVIG medication. All three patients were in remission prior to the last follow up. One patient who received RTX early in the course of the disease did not respond even after five months of treatment but was then successfully treated with CS, IVIG, and AZA (4).

Ashton et al. (14) described a case series of twenty patients with anti-HMGCR myopathy and a mean initial CK level of 7,189 U/L. RTX was initiated in five patients who failed to respond to prednisolone, MTX, AZA, and IVIG. Improvement was seen in two patients treated with RTX. Those two patients were also statin- naive younger patients, so results were similar to those reported by Landon-Cardinal et al. (4). There are a few other case series in which RTX was used to treat patients with refractory anti-HMGCR but it resulted in no benefit $(10,15)$.

The current patient improved slightly with oral steroids and according to tests but did not go into remission. AZA was used for few days and was stopped due to adverse reactions. RTX was ultimately started. The patient started responding within one month of treatment and went into complete remission within 3 months of initiation of RTX, with normal CK levels and a normal MRC grade; the patient has not relapsed.

\section{Conclusion}

RTX was effective in the current patient with refractory anti-HMGCR myopathy. The patient's response to RTX leads to questions like why statin-naïve patients respond better, why it does not beneficial all the patients if autoimmunity is the cause, and whether RTX can be used in the initial course of disease to minimize that course. In addition to a few cases reported in the literature, the current case highlights the need for a prospective randomized trial to confirm the effectiveness of RTX in anti-HMGCR myopathy.

\section{Funding: None.}

Conflict of Interest: The authors have no conflicts of interest to disclose.

\section{References}

1. Christopher-Stine L, Casciola-Rosen LA, Hong G, Chung T, Corse AM, Mammen AL. A novel autoantibody recognizing $200-\mathrm{kd}$ and $100-\mathrm{kd}$ proteins is associated with an immune-mediated necrotizing myopathy. Arthritis Rheum. 2010; 62:2757-2766.

2. Mohassel P, Mammen AL. Statin-associated autoimmune myopathy and anti-HMGCR autoantibodies. Muscle Nerve. 2013; 48:477-483.

3. Tiniakou E, Pinal-Fernandez I, Lloyd TE, Albayda J, Paik J, Werner JL, Parks CA, Casciola-Rosen L, Christopher-Stine L, Mammen AL. More severe disease and slower recovery in younger patients with anti3-hydroxy-3-methylglutaryl-coenzyme A reductaseassociated autoimmune myopathy. Rheumatology (Oxford). 2017; 56:787-794.

4. Landon-Cardinal O, Allenbach Y, Soulages A, Rigolet A, Hervier B, Champtiaux N, Monzani Q, Solé G, Benveniste $\mathrm{O}$. Rituximab in the treatment of refractory anti-HMGCR immune-mediated necrotizing myopathy. J Rheumatol. 2019; 46:623-627.

5. Selva-O'Callaghan A, Alvarado-Cardenas M, PinalFernández I, Trallero-Araguás E, Milisenda JC, Martínez MÁ, Marín A, Labrador-Horrillo M, Juárez C, GrauJunyent JM. Statin-induced myalgia and myositis: An update on pathogenesis and clinical recommendations. Expert Rev Clin Immunol. 2018; 14:215-224. 
6. Hamann PD, Cooper RG, McHugh NJ, Chinoy H. Statininduced necrotizing myositis - A discrete autoimmune entity within the "statin-induced myopathy spectrum." Autoimmun Rev. 2013; 12:1177-1181.

7. Dixit A, Abrudescu A. A case of atorvastatin-associated necrotizing autoimmune myopathy, mimicking idiopathic polymyositis. Case Rep Rheumatol. 2018; 2018:5931046.

8. Nichols L, Pfeifer K, Mammen AL, Shahnoor N, Konersman CG. An unusual case of statin-induced myopathy: Anti-HMGCoA necrotizing autoimmune myopathy. J Gen Intern Med. 2015; 30:1879-1883.

9. Khan NAJ, Khalid S, Ullah S, Malik MU, Makhoul S. Necrotizing autoimmune myopathy: A rare variant of idiopathic inflammatory myopathies. J Investig Med High Impact Case Rep. 2017; 5:2324709617709031.

10. Ramanathan S, Langguth D, Hardy TA, Garg N, Bundell C, Rojana-Udomsart A, Dale RC, Robertson T, Mammen AL, Reddel SW. Clinical course and treatment of antiHMGCR antibody-associated necrotizing autoimmune myopathy. Neurol Neuroimmunol Neuroinflamm. 2015; 2: e96

11. Aggarwal R, Moghadam-Kia S, Lacomis D, Malik A, Qi Z, Koontz D, Burlingame RW, Oddis CV. Anti-hydroxy3-methylglutaryl-coenzyme A reductase (anti-HMGCR) antibody in necrotizing myopathy: treatment outcomes, cancer risk, and role of autoantibody level. Scand J Rheumatol. 2020; 49:405-411.

12. Allenbach Y, Drouot L, Rigolet A, et al. Anti-HMGCR autoantibodies in European patients with autoimmune necrotizing myopathies: Inconstant exposure to statin. Medicine (Baltimore). 2014; 93:150-157.

13. Allenbach Y, Mammen AL, Benveniste O, Stenzel W; Immune-Mediated Necrotizing Myopathies Working Group. 224th ENMC International Workshop: Clinicosero-pathological classification of immune-mediated necrotizing myopathies Zandvoort, The Netherlands, 1416 October 2016. Neuromuscul Disord. 2018; 28:87-99.

14. Ashton C, Junckerstorff R, Bundell C, Hollingsworth $\mathrm{P}$, Needham M. Treatment and outcomes in necrotising autoimmune myopathy: An Australian perspective. Neuromuscul Disord. 2016; 26:734-740.

15. Grable-Esposito P, Katzberg HD, Greenberg SA, Srinivasan J, Katz J, Amato AA. Immune-mediated necrotizing myopathy associated with statins. Muscle Nerve. 2010; 41:185-190.

Received November 11, 2020; Revised March 12, 2021; Accepted March 22, 2021.

*Address correspondence to:

Sonia Gupta, Department of Internal Medicine, Creighton University, 7710 Mercy Road \#3000, Omaha, Nebraska 68124, USA.

E-mail: sgu27944@creighton.edu

Released online in J-STAGE as advance publication March 31, 2021. 